\title{
EDITORIAL
}

\author{
H. Irschik - M. Krommer • C. Marchioli • G. J. Weng • \\ M. Ostoja-Starzewski
}

\section{Films over topography: from creeping flow to linear stability, theory and experiments, a review}

Published online: 6 April 2018

(C) Springer-Verlag GmbH Austria, part of Springer Nature 2018

In 2015, the Editors of Acta Mechanica decided to start the Review and Perspective in Mechanics section, with the aim of providing coherent reviews on selected topics of broad interest. Solicited papers in this section are anticipated to depict a vivid image on the current state of the art, but also highlight emerging methodologies and discuss future research directions.

In the seventh article of this Review and Perspective in Mechanics series, we present the work authored by Professor Nuri Aksel and Dipl.-Phys. Mario Schörner, from the University of Bayreuth, Germany, on gravity-driven films flowing over topographies. The review starts by examining the impact of topography and inertia on these films, from creeping flow to inertial flow conditions. Then, the Authors switch from the direct problem to the inverse problem of identifying unknown flow quantities from an observed response of the free surface. An important section of the article is devoted to the stability of films flowing over different types of undulated inclines: Here, a comprehensive survey of both theoretical and experimental investigations is given, with specific attention to recent developments in this difficult and rapidly evolving area.

The present review provides an updated analysis of the physics that govern film flows over surface topographies and a detailed overview of the theoretical, numerical, and experimental challenges that must be faced. The Editors are confident that this review, with an organized and structured presentation of the subject and with a personal perspective on the future development goals, will be extremely useful for the community.

\author{
H. Irschik \\ Linz, Austria \\ M. Krommer \\ Vienna, Austria \\ C. Marchioli $(\varangle)$ \\ Udine, Italy \\ E-mail: cristian.marchioli@uniud.it \\ G. J. Weng \\ New Brunswick, NJ, USA \\ M. Ostoja-Starzewski \\ Urbana, IL, USA
}

